\title{
Erratum to: Impaired Social Decision-Making Mediates the Association Between ADHD and Social Problems
}

\author{
Kathryn L. Humphreys ${ }^{1}$. Chardeé A. Galán ${ }^{2} \cdot$ Nim Tottenham $^{3} \cdot$ Steve S. Lee $^{4}$
}

Published online: 22 March 2017

(C) Springer Science+Business Media New York 2017

Erratum to: J Abnorm Child Psychol (2016) 44:1023-1032

DOI 10.1007/s10802-015-0095-7

There was a typographical error in Fig. 2 (page 1028). The coefficient for the path between ADHD symptoms and CBCL Social Problems should be listed as 0.32 . The corrected figure is as shown below.

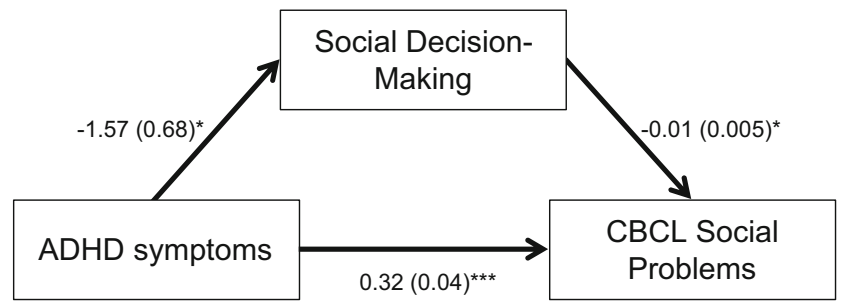

The online version of the original article can be found at http://dx.doi. org/10.1007/s10802-015-0095-7

Kathryn L. Humphreys

k.humphreys@stanford.edu

Chardeé A. Galán

chardeeashley@gmail.com

Nim Tottenham

nt17@columbia.edu

Steve S. Lee

steve.lee@psych.ucla.edu

1 Stanford University, Stanford, CA, USA

2 University of Pittsburgh, Pittsburgh, PA, USA

3 Columbia University, New York, NY, USA

4 University of California, Los Angeles, CA, USA 